Relations industrielles

Industrial Relations

\title{
The Growth of White-Collar Unionism, by George Sayers Bain, Don Mills, Ont., Oxford University Press, 1970, 233 pp.
}

\section{Jean Bernier}

Volume 26, numéro 2, 1971

URI : https://id.erudit.org/iderudit/028229ar

DOI : https://doi.org/10.7202/028229ar

Aller au sommaire du numéro

Éditeur(s)

Département des relations industrielles de l'Université Laval

ISSN

0034-379X (imprimé)

1703-8138 (numérique)

Découvrir la revue

Citer ce compte rendu

Bernier, J. (1971). Compte rendu de [The Growth of White-Collar Unionism, by George Sayers Bain, Don Mills, Ont., Oxford University Press, 1970, 233 pp.] Relations industrielles / Industrial Relations, 26(2), 510-511.

https://doi.org/10.7202/028229ar

Tous droits réservés @ C Département des relations industrielles de l'Université Laval, 1971
Ce document est protégé par la loi sur le droit d'auteur. L’utilisation des services d'Érudit (y compris la reproduction) est assujettie à sa politique d'utilisation que vous pouvez consulter en ligne.

https://apropos.erudit.org/fr/usagers/politique-dutilisation/ 
than detailed supervision of job conditions and control of the exact terms of employment. This arms-length relationship to the worker on the job may help explain the absence of industrial conflict and the ease with which most settlements are reached, and the modest role of the government in guidance of these relations particularly until such time as administration seeks to enforce economic controls. Even then the employer's selfinterest moves him to greater liberalism in action than the law or agreement prescribes and creates a level of industrial relations quite independent of the formal one. But the truncated Continental system appears to have run its course.

\section{Solomon BARKIN}

The Legal Status of Collective Agreements in England, the United States and Canada, by B.L. Adell, Kingston, Queen's University, Industrial Relations Center, 1970, xxxi et 240 pp.

Il s'agit d'une étude du caractère obligatoire de la convention collective en Angleterre, aux Etats-Unis et au Canada, selon le partage classique : effets «contractuels 》 entre les signataires et «normatifs » par rapport aux salariés. Chacun des trois tableaux trace la genèse du droit positif.

Jusqu'à présent du moins, dans le premier cas, la convention collective s'était tenue à l'écart du courant contractuel; si elle a une portée normative, ce ne pourrait être qu'en tant qu'usage ou coutume. $\mathrm{Au}$ contraire, aux EtatsUnis et au Canada, après maintes tentatives d'utiliser les techniques du droit commun, l'arbitrage, surtout, apporte les solutions. Beaucoup plus sûrement aux Etats-Unis, où la convention s'impose directement plutôt que par le biais du contrat individuel de travail. La théorie de l'incorporation du contenu de la convention dans ce dernier continue toujours en effet de mener une vie parallèle au Canada, ce qui ne peut qu'être prétexte à l'intervention du tribunal de droit commun. L'on doit par ailleurs examiner le rôle que peut jouer le salarié dans la mise à exécution de la convention, sans le concours du syndicat. Dans la mesure où le pouvoir de représentation de ce dernier y fait obstacle, doit s'affirmer l'obligation d'une représentation adéquate du salarié. Le Québec s'insère dans cet ensemble, comme on l'affirme d'ail- leurs, bien que l'on fasse relativement grand état du régime assez particulier de la convention collective étendue.

Pour éviter des absolus dangereux : l'ouvrage marque assurément une date importante dans le développement du droit du travail au Canada. Il évite la simple juxtaposition, souvent superficielle, des onze lois pour aller à la racine de l'ensemble du droit applicable, qu'il expose ensuite rigoureusement. Le recul de l'étude théorique de la convention collective, y compris l'examen critique de certaines données passées ou étrangères, permet à l'auteur de faire progresser la solution des principaux problèmes pratiques qui s'attachent, d'un point de vue juridique, à la mise à exécution forcée de la convention collective.

Pierre VERGE

The Growth of White-Collar Unionism, by George Sayers Bain, Don Mills, Ont., Oxford University Press, 1970, 233 pp.

L'une des caractéristiques du développement des économies modernes réside dans l'accroissement considérable aussi bien en termes absolus qu'en termes relatifs du nombre de cols-blancs par rapport aux cols-bleus. Cette évolution se produit à un rythme tel que le nombre des cols-blancs dépassera bientôt celui des cols-bleus dans la plupart des pays industrialisés où ce n'est pas déjà fait.

Cette situation soulève un certain nombre de questions quant au développement du syndicalisme dans l'avenir car si les syndicats n'arrivent pas à rejoindre ces travailleurs, ils sont condamnés à occuper une place de plus en plus marginale au sein de la main-d'œuvre active.

C'est à quelques-uns des problèmes posés au syndicalisme par ce changement dans la structure occupationnelle que l'auteur, George Sayers Bain, s'intéresse dans cet ouvrage qui est le résultat d'une recherche qui a duré quatre années. Il a tenté en effet de découvrir quels sont les principaux facteurs qui favorisent le développement du syndicalisme chez les cols-blancs en Grande-Bretagne et en particulier dans l'industrie manufacturière.

Après avoir étudié l'importance des cols-blancs dans la main-d'œuvre anglaise, l'auteur fait état de la situation syndicale dans ce secteur. 
Puis il procède à l'analyse en profondeur de certains facteurs susceptibles d'expliquer la croissance des syndicats chez les cols-blancs. A cet égard, il tient compte notamment des caractéristiques socio-démographiques de ces travailleurs, de leur situation économique et de leur place dans le monde du travail. Il considère également le rôle des syndicats, des employeurs et du gouvernement ainsi que l'effet de la situation sociale sur le développement de ce type de syndicalisme.

Il faut dire que cette recherche, en plus d'être appuyée sur une base théorique bien documentée, dépasse les considérations à caractère purement déductif dans un effort de vérification au niveau empirique.

C'est ainsi que l'auteur en est arrivé à la conclusion que la croissance du syndicalisme chez les cols-blancs est reliée au degré de concentration de ce type de main-d'œuvre, à l'attitude des employeurs eu égard à la reconnaissance de tels syndicats et à l'action gouvernementale en cette matière.

Enfin, il faut dire qu'en plus de l'intérêt que comporte cette étude par l'éclairage qu'elle apporte sur ce problème très actuel, elle présente de nombreuses considérations d'ordre méthodologique qui permettent de suivre la démarche de l'auteur.

Jean BERNIER

Labor-Management Relations in the $\mathrm{Pu}$ blic Service, by Harold S. Roberts, Honolulu, Hawaii, University of Hawaii Press, 1970,617 pp.

Depuis la fin de la dernière guerre, les relations de travail dans les services publics se sont considérablement développées. Ceci tient à plusieurs causes: gonflement du secteur lui-même, multiplication du nombre des emplois et, par ricochet, désir plus intense des travailleurs de recourir au syndicalisme comme moyen de défense et de promotion de leurs droits et de leurs intérêts. Il ne s'est pas agi là d'un phénomène propre à la province de Québec et au Canada. On l'observe également aux Etats-Unis pendant la même période.

Aussi le gouvernement de Washington, ceux des Etats, des municipalités et des conseils scolaires ont-ils dû se mettre à l'œuvre pour découvrir les moyens les mieux appropriés pour y faire face. Mais se retrouver dans cette masse d'études, de rapports d'enquête, de lois et de règlements, c'est une entreprise quasi impossible à moins de ne pouvoir y consacrer des semaines et des mois. La dernière édition d'un volumineux ouvrage d'Harold Roberts, décédé il y a un peu plus d'un an, comble-t-elle dans une bonne mesure cette lacune? Il s'agit d'ailleurs d'une cinquième édition, chaque fois revue et corrigée, enrichie chaque fois d'éléments nouveaux acquis dans la pratique quotidienne des relations de travail aux divers niveaux de gouvernement.

Un semblable ouvrage, qui constitue en quelque sorte un compte rendu exhaustif de ce qu'on a appelé en certains milieux «la révolution de l'employé du secteur public », ne se résume pas.

Le volume est divisé en onze chapitres qui traitent de la plupart des sujets relatifs aux relations de travail dans les multiples services publics que sont le gouvernement fédéral, ceux des Etats ainsi que des nombreuses agences qui gravitent autour d'eux. L'ouvrage comprend notamment une analyse de la législation en vigueur à Washington, législation instaurée par Kennedy et modifiée plus tard sous le président Nixon, ainsi que celle qui existe dans la plupart des Etats, une étude des critères utilisés dans la détermination des unités de négociation dans les services publics, un exposé des mécanismes variés institués en vue du règlement des griefs, qu'il s'agisse de griefs soulevés par les employés pris individuellement ou de griefs découlant de l'application ou de l'interprétation des conventions cellectives de travail, une analyse des moyens destinés à prévenir et à résoudre les conflits.

Le huitième chapitre est d'un intérêt particulier en ce qu'il reproduit textuellement une trentaine de conventions collectives. A titre d'exemple, en parcourant ces textes, on y trouve dans une convention intervenue entre le conseil scolaire de la ville d'Huntingdon et l'Association des enseignants de cette même ville deux articles intéressants : l'un traitant de la protection des droits des instituteurs et l'autre de la liberté académique.

Faut-il signaler aussi le premier chapitre ou l'Auteur, qui a déjà publié un dictionnaire des relations du travail, pré- 